\title{
Half-discrete Hilbert-type inequalities involving differential operators
}

\author{
Vandanjav Adiyasuren ${ }^{1}$, Tserendorj Batbold ${ }^{2 *}$ and Mario Krnić ${ }^{3}$
}

${ }^{\text {*} C o r r e s p o n d e n c e: ~}$

tsbatbold@hotmail.com

${ }^{2}$ Institute of Mathematics, National University of Mongolia, P.O. Box 46A/104, Ulaanbaatar, 14201, Mongolia

Full list of author information is available at the end of the article

\begin{abstract}
Motivated by some recent results, in this paper we derive several half-discrete Hilbert-type inequalities with a homogeneous kernel involving some differential operators. The main results are proved for the case of non-conjugate parameters. After reduction to the conjugate case, we show that the constants appearing on the right-hand sides of these inequalities are the best possible.
\end{abstract}

MSC: Primary 26D10; 26D15; secondary 33B15

Keywords: Hilbert inequality; Hardy inequality; half-discrete inequality; differential operator; homogeneous function

\section{Introduction}

The Hilbert inequality is one of the most interesting inequalities in mathematical analysis and its applications. Although classical, it is still of interest to numerous authors.

Recently, Azar [1], obtained a new form of the Hilbert inequality including a differential operator. In order to state this result and to summarize our further discussion, we start by giving some notation. We denote by $\mathcal{D}_{+}^{m}, m \geq 0$, a differential operator defined by $\mathcal{D}_{+}^{m} f(x)=$ $f^{(m)}(x)$, where $f^{(m)}$ stands for the $m$ th derivative of a function $f: \mathbb{R}_{+} \rightarrow \mathbb{R}$. In addition, throughout this article, $\Lambda_{+}^{m}$ is the set of non-negative measurable functions $f: \mathbb{R}_{+} \rightarrow \mathbb{R}$ such that $f^{(m)}$ exists a.e. on $\mathbb{R}_{+}, f^{(m)}(x)>0$, a.e. on $\mathbb{R}_{+}$, and $f^{(k)}(0)=0, k=0,1,2, \ldots, m-1$.

Now, a differential form of the Hilbert inequality derived in [1] can be stated as follows: Let $p$ and $q$ be non-negative conjugate parameters, i.e. $\frac{1}{p}+\frac{1}{q}=1, p>1$, let $p m<s \leq q$, where $m$ is a fixed non-negative integer, and let $C=\frac{\Gamma\left(\frac{s}{p}-m\right) \Gamma\left(\frac{s}{q}\right)}{\Gamma(s)}$, where $\Gamma$ stands for a usual Gamma function. Then the inequality

$$
\int_{0}^{\infty} f(x) \sum_{n=1}^{\infty} \frac{a_{n}}{(x+n)^{s}} d x<C\left[\int_{0}^{\infty} x^{p(m+1)-s-1}\left(\mathcal{D}_{+}^{m} f(x)\right)^{p} d x\right]^{\frac{1}{p}}\left[\sum_{n=1}^{\infty} n^{q-s-1} a_{n}^{q}\right]^{\frac{1}{q}}
$$

holds for all $f \in \Lambda_{+}^{m}, f \neq 0$, and for all non-negative sequences $a=\left(a_{n}\right)_{n \in \mathbb{N}}, a \neq 0$, provided that the integral and the series on the right-hand side converge. Moreover, the constant $C$ is the best possible in the sense that it cannot be replaced with a smaller constant so that (1) still holds for all $f \in \Lambda_{+}^{m}$ and for all non-negative sequences $a=\left(a_{n}\right)_{n \in \mathbb{N}}$. The above inequality may be regarded as an extension of a classical Hilbert inequality since for $m=0$, $p=q=2$, and $s=1$, we obtain the non-weighted inequality with the previously known sharp constant $C=\pi$ (for more details, see [2]). For a comprehensive study of an initial development of the Hilbert inequality, the reader is referred to a classical monograph [2]. 
It should be noticed here that the inequality (1) is usually referred to as a half-discrete Hilbert-type inequality since it includes both integral and a sum.

The main objective of this paper is to extend the half-discrete inequality (1) to hold for a general homogeneous function. Recall that a function $K: \mathbb{R}_{+} \times \mathbb{R}_{+} \rightarrow \mathbb{R}$ is said to be homogeneous of degree $-s, s>0$, if $K(t x, t y)=t^{-s} K(x, y)$, for every $x, y, t \in \mathbb{R}_{+}$. In addition, for such a function we define

$$
k(\eta)=\int_{0}^{\infty} K(1, t) t^{-\eta} d t
$$

If nothing else is explicitly stated, we assume that the integral $k(\eta)$ converges for considered values of $\eta$.

On the other hand, the recent paper [3] provides a unified treatment of half-discrete Hilbert-type inequalities with a homogeneous kernel and in the setting with nonconjugate exponents.

Suppose $p$ and $q$ are real parameters, such that

$$
p>1, \quad q>1, \quad \frac{1}{p}+\frac{1}{q} \geq 1
$$

and let $p^{\prime}=\frac{p}{p-1}$ and $q^{\prime}=\frac{q}{q-1}$ respectively be their conjugate exponents, that is, $\frac{1}{p}+\frac{1}{p^{\prime}}=1$ and $\frac{1}{q}+\frac{1}{q^{\prime}}=1$. Further, define

$$
\lambda=\frac{1}{p^{\prime}}+\frac{1}{q^{\prime}}
$$

and observe that $0<\lambda \leq 1$ holds for all $p$ and $q$ as in (2). In particular, equality $\lambda=1$ holds in (3) if and only if $q=p^{\prime}$, that is, only if $p$ and $q$ are mutually conjugate. Otherwise, we have $0<\lambda<1$, and such parameters $p$ and $q$ will be referred to as non-conjugate exponents.

In the above setting with non-conjugate exponents and with a homogeneous kernel $K$ of degree $-s, s>0$, Krnić et al. [3], showed that the following pair of equivalent half-discrete Hilbert-type inequalities:

$$
\begin{aligned}
& \sum_{n=1}^{\infty} a_{n} \int_{0}^{\infty} K^{\lambda}(x, n) f(x) d x \\
& \quad=\int_{0}^{\infty} f(x)\left(\sum_{n=1}^{\infty} K^{\lambda}(x, n) a_{n}\right) d x \\
& \quad<L\left[\int_{0}^{\infty} x^{\frac{p}{q^{f^{\prime}}}(1-s)+p\left(\alpha_{1}-\alpha_{2}\right)} f^{p}(x) d x\right]^{\frac{1}{p}}\left[\sum_{n=1}^{\infty} n^{\frac{q}{p^{\prime}}(1-s)+q\left(\alpha_{2}-\alpha_{1}\right)} a_{n}^{q}\right]^{\frac{1}{q}}
\end{aligned}
$$

and

$$
\begin{aligned}
& {\left[\sum_{n=1}^{\infty} n^{\frac{q^{\prime}}{p^{\prime}}(s-1)+q^{\prime}\left(\alpha_{1}-\alpha_{2}\right)}\left(\int_{0}^{\infty} K^{\lambda}(x, n) f(x) d x\right)^{q^{\prime}}\right]^{\frac{1}{q^{\prime}}}} \\
& <L\left[\int_{0}^{\infty} x^{\frac{p}{q^{\prime}}(1-s)+p\left(\alpha_{1}-\alpha_{2}\right)} f^{p}(x) d x\right]^{\frac{1}{p}}
\end{aligned}
$$


holds for any non-negative measurable function $f: \mathbb{R}_{+} \rightarrow \mathbb{R}$ and a non-negative sequence $a=\left(a_{n}\right)_{n \in \mathbb{N}}$, where $L=k^{\frac{1}{q^{\prime}}}\left(q^{\prime} \alpha_{2}\right) k^{\frac{1}{p^{\prime}}}\left(2-s-p^{\prime} \alpha_{1}\right)$, and $\alpha_{1}, \alpha_{2}$ are real parameters such that the function $K(x, y) y^{-q^{\prime} \alpha_{2}}$ is decreasing on $\mathbb{R}_{+}$for any $x \in \mathbb{R}_{+}$. Clearly, in the above inequalities all integrals and sums are assumed to be convergent, and the function and the sequence are not equal to zero. Inequalities (4) and (5) will be an important tool in our extension of (1) to a general homogeneous case. For some particular half-discrete inequalities and related results, the reader is referred to recent papers [4-8] and references therein. Moreover, for comprehensive accounts on Hilbert inequality including history, different proofs, refinements and diverse applications, we refer to recent monograph [9].

The paper is divided into four sections as follows: After this Introduction, in Section 2, we cite several auxiliary results needed for our study. Further, in Section 3 we give several extensions of inequality (1) to a general homogeneous case and in the setting with non-conjugate parameters. It should be noticed here that our methods of proving differ from the techniques presented in [1]. After reduction to conjugate case, in Section 4, we establish conditions for which derived inequalities include the best constants on their right-hand sides.

\section{Notation and auxiliary results}

Throughout this paper $\Gamma(\cdot)$ stands for the usual Gamma function, that is,

$$
\Gamma(a)=\int_{0}^{\infty} t^{a-1} e^{-t} d t, \quad a>0,
$$

while $B(\cdot, \cdot)$ denotes the usual Beta function, i.e.

$$
B(a, b)=\int_{0}^{1} t^{a-1}(1-t)^{b-1} d t, \quad a, b>0 .
$$

The basic relationship between the Beta and the Gamma function is given by

$$
B(a, b)=\frac{\Gamma(a) \Gamma(b)}{\Gamma(a+b)}, \quad a, b>0 .
$$

The rising factorial power $x^{\bar{m}}$, where $m$ is a non-negative integer, also known as a Pochhammer symbol, is defined by

$$
x^{\bar{m}}=x(x+1)(x+2) \cdots(x+m-1)
$$

while the falling factorial power $x^{\underline{\underline{m}}}$ is given by

$$
x^{\underline{\underline{m}}}=x(x-1)(x-2) \cdots(x-m+1) .
$$

The rising and falling factorial powers can be expressed in terms of the usual Gamma function, i.e.

$$
x^{\bar{m}}=\frac{\Gamma(x+m)}{\Gamma(x)} \quad \text { and } \quad x^{\underline{m}}=\frac{\Gamma(x+1)}{\Gamma(x-m+1)} .
$$


It should be noticed here that the above relations hold for complex arguments of the Gamma function which are not negative integers (for more details, see e.g. [10] or [11]).

In contrast to the techniques of proving in [1], our results will be established by virtue of the famous Hardy inequality. In 1928, Hardy [12] proved an estimate for the integration operator (or the Hardy operator)

$$
\mathcal{H} f(x)=\int_{0}^{x} f(t) d t
$$

from which the first weighted modification of the Hardy inequality followed, namely the inequality

$$
\int_{0}^{\infty} x^{-r}(\mathcal{H} f(x))^{p} d x<\left(\frac{p}{r-1}\right)^{p} \int_{0}^{\infty} x^{p-r} f^{p}(x) d x
$$

valid with $p>1, r>1,0<\int_{0}^{\infty} x^{p-r} f^{p}(x) d x<\infty$, where the constant $\left(\frac{p}{r-1}\right)^{p}$ is the best possible (for more details, see [2], Theorem 330, and [13]). A dual Hardy inequality, accompanied by a dual integration operator or dual Hardy operator

$$
\mathcal{H}^{*} f(x)=\int_{x}^{\infty} f(t) d t
$$

asserts that

$$
\int_{0}^{\infty} x^{-r}\left(\mathcal{H}^{*} f(x)\right)^{p} d x<\left(\frac{p}{1-r}\right)^{p} \int_{0}^{\infty} x^{p-r} f^{p}(x) d x,
$$

holds for $p>1$ and $r<1$, provided that $0<\int_{0}^{\infty} x^{p-r} f^{p}(x) d x<\infty$ (for more details, see [13]).

\section{Half-discrete inequalities in the non-conjugate case}

Our first intention is to give an extension of inequality (1) to the case of non-conjugate exponents and a general homogeneous function. Having in mind relations (4) and (5), our results will be given in two equivalent forms. It is interesting that the constants appearing in our extended inequalities are also expressed in terms of the Gamma function.

Theorem 1 Let $p, q, p^{\prime}, q^{\prime}$, and $\lambda$ be as in (2) and (3), and let $K: \mathbb{R}_{+} \times \mathbb{R}_{+} \rightarrow \mathbb{R}$ be a non-negative measurable homogeneous function of degree $-s, s>0$. If $\alpha_{1}$ and $\alpha_{2}$ are real parameters such that the function $K(x, y) y^{-q^{\prime} \alpha_{2}}$ is decreasing on $\mathbb{R}_{+}$for any fixed $x \in \mathbb{R}_{+}$ and $\beta:=\frac{1}{q^{\prime}}(s-1)+\alpha_{2}-\alpha_{1}-\frac{1}{p}>m-1$, where $m$ is a fixed non-negative integer, then the inequalities

$$
\begin{aligned}
& \sum_{n=1}^{\infty} a_{n} \int_{0}^{\infty} K^{\lambda}(x, n) f(x) d x \\
& \quad=\int_{0}^{\infty} f(x)\left(\sum_{n=1}^{\infty} K^{\lambda}(x, n) a_{n}\right) d x \\
& \quad<L \cdot \frac{\Gamma(\beta-m+1)}{\Gamma(\beta+1)}\left[\int_{0}^{\infty} x^{p(m-\beta)-1}\left(\mathcal{D}_{+}^{m} f(x)\right)^{p} d x\right]^{\frac{1}{p}}\left[\sum_{n=1}^{\infty} n^{\frac{q}{p^{\prime}}(1-s)+q\left(\alpha_{2}-\alpha_{1}\right)} a_{n}^{q}\right]^{\frac{1}{q}}
\end{aligned}
$$


and

$$
\begin{aligned}
& {\left[\sum_{n=1}^{\infty} n^{\frac{q^{\prime}}{p^{\prime}}(s-1)+q^{\prime}\left(\alpha_{1}-\alpha_{2}\right)}\left(\int_{0}^{\infty} K^{\lambda}(x, n) f(x) d x\right)^{q^{\prime}}\right]^{\frac{1}{q^{\prime}}}} \\
& <L \cdot \frac{\Gamma(\beta-m+1)}{\Gamma(\beta+1)}\left[\int_{0}^{\infty} x^{p(m-\beta)-1}\left(\mathcal{D}_{+}^{m} f(x)\right)^{p} d x\right]^{\frac{1}{p}},
\end{aligned}
$$

where $0<L:=k^{\frac{1}{q^{\prime}}}\left(q^{\prime} \alpha_{2}\right) k^{\frac{1}{p^{\prime}}}\left(2-s-p^{\prime} \alpha_{1}\right)<\infty$, hold for a non-negative function $f \in \Lambda_{+}^{m}$ and a non-negative sequence $a=\left(a_{n}\right)_{n \in \mathbb{N}}$, provided that the integral and series on their right-hand sides converge to positive numbers.

Proof Clearly, if $m=0$, inequalities (8) and (9) coincide with (4) and (5), respectively. Otherwise, rewrite the right-hand side of (4) in a form that is more suitable for the application of the Hardy inequality. Namely, since

$$
\mathcal{H}\left(\mathcal{D}_{+} f\right)(x)=\int_{0}^{x} f^{\prime}(t) d t=f(x)-f(0)=f(x)
$$

we have

$$
\left[\int_{0}^{\infty} x^{\frac{p}{q^{(}(1-s)+p\left(\alpha_{1}-\alpha_{2}\right)}} f^{p}(x) d x\right]^{\frac{1}{p}}=\left[\int_{0}^{\infty} x^{-p \beta-1}\left(\mathcal{H}\left(\mathcal{D}_{+} f\right)(x)\right)^{p} d x\right]^{\frac{1}{p}} .
$$

Moreover, due to the weighted Hardy inequality (6), it follows that

$$
\left[\int_{0}^{\infty} x^{-p \beta-1}\left(\mathcal{H}\left(\mathcal{D}_{+} f\right)(x)\right)^{p} d x\right]^{\frac{1}{p}}<\frac{1}{\beta}\left[\int_{0}^{\infty} x^{p(1-\beta)-1}\left(\mathcal{D}_{+} f(x)\right)^{p} d x\right]^{\frac{1}{p}} .
$$

Now, by applying the Hardy inequality to the right-hand side of the last inequality $m-1$ times, we get the relation

$$
\left[\int_{0}^{\infty} x^{-p \beta-1}\left(\mathcal{H}\left(\mathcal{D}_{+} f\right)(x)\right)^{p} d x\right]^{\frac{1}{p}}<\frac{1}{\beta^{\underline{m}}}\left[\int_{0}^{\infty} x^{p(m-\beta)-1}\left(\mathcal{D}_{+}^{m} f(x)\right)^{p} d x\right]^{\frac{1}{p}} .
$$

Finally, the inequality (8) holds due to (4), (10), and (11). In the same way the inequality (9) follows by virtue of (5) and (11), which completes the proof.

The previous theorem is derived by virtue of the Hardy inequality and covers the case when $\beta>m-1$, where $m$ is a fixed non-negative integer. Our next result is in some way complementary to Theorem 1 since it covers the case when $\beta<0$. The crucial step in proving the corresponding relations will be the dual Hardy inequality (7).

In order to state this result, we define a differential operator $\mathcal{D}_{ \pm}^{m}$ by $\mathcal{D}_{ \pm}^{m} f(x)=(-1)^{m} \times$ $f^{(m)}(x)$, where $m$ is a non-negative integer. Moreover, the following theorem holds for all non-negative functions $f: \mathbb{R}_{+} \rightarrow \mathbb{R}$ such that the $m$ th derivative $f^{(m)}$ exists a.e. on $\mathbb{R}_{+}$, $\mathcal{D}_{ \pm}^{k} f(x)>0, k=0,1,2, \ldots, m$, a.e. on $\mathbb{R}_{+}$, and $\lim _{x \rightarrow \infty} f^{(k)}(x)=0$ for $k=0,1,2, \ldots, m-1$. This set of functions will be denoted by $\Lambda_{ \pm}^{m}$. 
Theorem 2 Let $p, q, p^{\prime}, q^{\prime}$, and $\lambda$ be as in (2) and (3), and let $K: \mathbb{R}_{+} \times \mathbb{R}_{+} \rightarrow \mathbb{R}$ be a nonnegative measurable homogeneous function of degree $-s, s>0$. Further, let $\alpha_{1}$ and $\alpha_{2}$ be real parameters such that the function $K(x, y) y^{-q^{\prime} \alpha_{2}}$ is decreasing on $\mathbb{R}_{+}$for any fixed $x \in \mathbb{R}_{+}$and $\beta:=\frac{1}{q^{\prime}}(s-1)+\alpha_{2}-\alpha_{1}-\frac{1}{p}<0$. If $0<L:=k^{\frac{1}{q^{\prime}}}\left(q^{\prime} \alpha_{2}\right) k^{\frac{1}{p^{\prime}}}\left(2-s-p^{\prime} \alpha_{1}\right)<\infty$, then the inequalities

$$
\begin{aligned}
& \sum_{n=1}^{\infty} a_{n} \int_{0}^{\infty} K^{\lambda}(x, n) f(x) d x \\
& \quad=\int_{0}^{\infty} f(x)\left(\sum_{n=1}^{\infty} K^{\lambda}(x, n) a_{n}\right) d x \\
& \quad<L \cdot \frac{\Gamma(-\beta)}{\Gamma(-\beta+m)}\left[\int_{0}^{\infty} x^{p(m-\beta)-1}\left(\mathcal{D}_{ \pm}^{m} f(x)\right)^{p} d x\right]^{\frac{1}{p}}\left[\sum_{n=1}^{\infty} n^{\frac{q}{p^{\prime}}(1-s)+q\left(\alpha_{2}-\alpha_{1}\right)} a_{n}^{q}\right]^{\frac{1}{q}}
\end{aligned}
$$

and

$$
\begin{aligned}
& {\left[\sum_{n=1}^{\infty} n^{\frac{q^{\prime}}{p^{\prime}}(s-1)+q^{\prime}\left(\alpha_{1}-\alpha_{2}\right)}\left(\int_{0}^{\infty} K^{\lambda}(x, n) f(x) d x\right)^{q^{\prime}}\right]^{\frac{1}{q^{\prime}}}} \\
& \quad<L \cdot \frac{\Gamma(-\beta)}{\Gamma(-\beta+m)}\left[\int_{0}^{\infty} x^{p(m-\beta)-1}\left(\mathcal{D}_{ \pm}^{m} f(x)\right)^{p} d x\right]^{\frac{1}{p}}
\end{aligned}
$$

hold for any non-negative function $f \in \Lambda_{ \pm}^{m}$ and a non-negative sequence $a=\left(a_{n}\right)_{n \in \mathbb{N}}$, provided that the integral and series on their right-hand sides converge to positive numbers.

Proof We follow the same procedure as in the proof of Theorem 1, this time accompanied by the dual Hardy inequality (7). We have

$$
\left[\int_{0}^{\infty} x^{\frac{p}{q^{(}}(1-s)+p\left(\alpha_{1}-\alpha_{2}\right)} f^{p}(x) d x\right]^{\frac{1}{p}}=\left[\int_{0}^{\infty} x^{-p \beta-1}\left(\mathcal{H}^{*}\left(\mathcal{D}_{ \pm} f\right)(x)\right)^{p} d x\right]^{\frac{1}{p}}
$$

since

$$
\mathcal{H}^{*}\left(\mathcal{D}_{ \pm} f\right)(x)=-\int_{x}^{\infty} f^{\prime}(t) d t=f(x)
$$

Moreover, utilizing the dual Hardy inequality $m$ times, it follows that

$$
\left[\int_{0}^{\infty} x^{-p \beta-1}\left(\mathcal{H}^{*}\left(\mathcal{D}_{ \pm} f\right)(x)\right)^{p} d x\right]^{\frac{1}{p}}<\frac{1}{(-\beta)^{\bar{m}}}\left[\int_{0}^{\infty} x^{p(m-\beta)-1}\left(\mathcal{D}_{ \pm}^{m} f(x)\right)^{p} d x\right]^{\frac{1}{p}}
$$

Now, the relations (4), (14), and (15) entail the desired inequality (12). Similarly, the inequality (13) follows by virtue of (5) and (15).

Remark 1 It should be noticed here that Theorem 1 and Theorem 2 coincide in the case of $m=0$. Therefore, presented results may be regarded as the differential extensions of inequalities (4) and (5).

We have already mention that the problem of the best constants is one of the most interesting questions in connection with Hilbert-type inequalities. Unfortunately, there is still 
no evidence that the constants appearing on the right-hand sides of relations (8), (9), (12), and (13) are the best possible. This problem seems to be very hard in the non-conjugate case and remains still open. Luckily, we can solve the mentioned problem for some particular settings in the conjugate case.

\section{Reduction to conjugate case and the best constants}

Now, our goal is to determine conditions under which the constants appearing on the right-hand sides of inequalities (8), (9), (12), and (13) are the best possible.

Therefore, in this section we deal with non-negative conjugate exponents $p, q$, that is, with parameters $p$ and $q$ such that $\frac{1}{p}+\frac{1}{q}=1, p>1$. In this case $p^{\prime}=q, q^{\prime}=p$, and $\lambda=1$.

It should be noticed here that the constant appearing in the inequality (1) does not contain any exponent. Keeping in mind this fact, we are going to simplify the constants appearing in (8), (9), (12), and (13) so that they do not contain exponents. Hence, it is natural to impose the condition

$$
p \alpha_{2}+q \alpha_{1}=2-s
$$

since in this case relation $k\left(p \alpha_{2}\right)=k\left(2-s-q \alpha_{1}\right)$ holds. With this assumption, the constant $L$ appearing in Theorem 1 and Theorem 2 reduces to $L^{*}=k\left(p \alpha_{2}\right)$.

Thus, if the condition (16) is fulfilled, the conjugate forms of inequalities (8) and (9) become, respectively,

$$
\begin{aligned}
\sum_{n=1}^{\infty} a_{n} \int_{0}^{\infty} K(x, n) f(x) d x \\
\quad=\int_{0}^{\infty} f(x)\left(\sum_{n=1}^{\infty} K(x, n) a_{n}\right) d x \\
\quad<L^{*} \cdot \frac{\Gamma(\beta-m+1)}{\Gamma(\beta+1)}\left[\int_{0}^{\infty} x^{p(m-\beta)-1}\left(\mathcal{D}_{+}^{m} f(x)\right)^{p} d x\right]^{\frac{1}{p}}\left[\sum_{n=1}^{\infty} n^{-1+p q \alpha_{2}} a_{n}^{q}\right]^{\frac{1}{q}}
\end{aligned}
$$

and

$$
\begin{aligned}
& {\left[\sum_{n=1}^{\infty} n^{(p-1)\left(1-p q \alpha_{2}\right)}\left(\int_{0}^{\infty} K(x, n) f(x) d x\right)^{p}\right]^{\frac{1}{p}}} \\
& \quad<L^{*} \cdot \frac{\Gamma(\beta-m+1)}{\Gamma(\beta+1)}\left[\int_{0}^{\infty} x^{p(m-\beta)-1}\left(\mathcal{D}_{+}^{m} f(x)\right)^{p} d x\right]^{\frac{1}{p}},
\end{aligned}
$$

where $\beta=-q \alpha_{1}$. In the same setting, inequalities (12) and (13) read, respectively,

$$
\begin{aligned}
& \sum_{n=1}^{\infty} a_{n} \int_{0}^{\infty} K(x, n) f(x) d x \\
& \quad=\int_{0}^{\infty} f(x)\left(\sum_{n=1}^{\infty} K(x, n) a_{n}\right) d x \\
& \quad<L^{*} \cdot \frac{\Gamma(-\beta)}{\Gamma(-\beta+m)}\left[\int_{0}^{\infty} x^{p(m-\beta)-1}\left(\mathcal{D}_{ \pm}^{m} f(x)\right)^{p} d x\right]^{\frac{1}{p}}\left[\sum_{n=1}^{\infty} n^{-1+p q \alpha_{2}} a_{n}^{q}\right]^{\frac{1}{q}}
\end{aligned}
$$


and

$$
\begin{aligned}
& {\left[\sum_{n=1}^{\infty} n^{(p-1)\left(1-p q \alpha_{2}\right)}\left(\int_{0}^{\infty} K(x, n) f(x) d x\right)^{p}\right]^{\frac{1}{p}}} \\
& \quad<L^{*} \cdot \frac{\Gamma(-\beta)}{\Gamma(-\beta+m)}\left[\int_{0}^{\infty} x^{p(m-\beta)-1}\left(\mathcal{D}_{ \pm}^{m} f(x)\right)^{p} d x\right]^{\frac{1}{p}},
\end{aligned}
$$

where $\beta=-q \alpha_{1}$.

Remark 2 Let $K(x, y)=(x+y)^{-s}, s>0$, and $\alpha_{1}=\frac{p-s}{p q}, \alpha_{2}=\frac{q-s}{p q}$. In this case the constant $L^{*}$ appearing in inequalities (17), (18), (19), and (20) is expressed in terms of the Beta function, that is, $L^{*}=k\left(1-\frac{s}{q}\right)=B\left(\frac{s}{p}, \frac{s}{q}\right)$. Then, utilizing the relationship between the Beta and the Gamma functions, we have $L^{*} \cdot \frac{\Gamma(\beta-m+1)}{\Gamma(\beta+1)}=\frac{\Gamma\left(\frac{s}{p}-m\right) \Gamma\left(\frac{s}{q}\right)}{\Gamma(s)}$, that is, the relation (17) becomes the inequality (1) from the Introduction, with a weaker condition $p m<s$. Thus, the dual form of (1) includes the constant which reduces to $L^{*} \cdot \frac{\Gamma(-\beta)}{\Gamma(-\beta+m)}=\frac{\pi}{\sin \frac{s \pi}{p}} \cdot \frac{\Gamma\left(\frac{s}{q}\right)}{\Gamma(s) \Gamma\left(m+1-\frac{s}{p}\right)}$, after applying the Euler reflection formula $\Gamma(x) \Gamma(1-x)=\frac{\pi}{\sin x \pi}$.

Now, our aim is to show that the constants appearing in (17), (18), (19), and (20) are the best possible. The corresponding proofs are the substance of the following two theorems.

Theorem 3 Let $p, q>1$ be conjugate parameters and $K: \mathbb{R}_{+} \times \mathbb{R}_{+} \rightarrow \mathbb{R}$ be a non-negative measurable homogeneous function of degree $-s, s>0$. Further, let $\alpha_{1}$ and $\alpha_{2}$ be real parameters fulfilling condition (16) and $\beta=-q \alpha_{1} \in(m-1, s-1), s>m$, where $m$ is a fixed non-negative integer. If the function $K(x, y) y^{-p \alpha_{2}}$ is decreasing on $\mathbb{R}_{+}$for any fixed $x \in \mathbb{R}_{+}$, then the constant $L^{*} \cdot \frac{\Gamma(\beta-m+1)}{\Gamma(\beta+1)}$ is the best possible in (17) and (18).

Proof In order to prove that the inequality (17) includes the best constant on its right-hand side, suppose that there exists a positive constant $C_{1}$, smaller than $L^{*} \cdot \frac{\Gamma(\beta-m+1)}{\Gamma(\beta+1)}$, such that the relation

$$
\begin{aligned}
& \sum_{n=1}^{\infty} a_{n} \int_{0}^{\infty} K(x, n) f(x) d x \\
& \quad=\int_{0}^{\infty} f(x)\left(\sum_{n=1}^{\infty} K(x, n) a_{n}\right) d x \\
& \quad<C_{1}\left[\int_{0}^{\infty} x^{p(m-\beta)-1}\left(\mathcal{D}_{+}^{m} f(x)\right)^{p} d x\right]^{\frac{1}{p}}\left[\sum_{n=1}^{\infty} n^{-1+p q \alpha_{2}} a_{n}^{q}\right]^{\frac{1}{q}}
\end{aligned}
$$

holds for any non-negative function $f \in \Lambda_{+}^{m}$ and a non-negative sequence $a=\left(a_{n}\right)_{n \in \mathbb{N}}$, provided that the integral and series on its right-hand side converge.

Now, let $\widetilde{L}$ and $\widetilde{R}$, respectively, denote the left-hand side and the right-hand side of (21) accompanied with

$$
\tilde{f}(x)=\frac{\Gamma\left(1+\beta-\frac{\varepsilon}{p}-m\right)}{\Gamma\left(1+\beta-\frac{\varepsilon}{p}\right)} \cdot x^{\beta-\frac{\varepsilon}{p}} \cdot \chi_{[1, \infty)}(x) \text { and } \quad \tilde{a}_{n}=n^{-p \alpha_{2}-\frac{\varepsilon}{q}}
$$


where $\varepsilon>0$ is a sufficiently small number. Here, $\chi$ stands for a characteristic function of the corresponding set. Since the $m$ th derivative of the function $x^{\beta-\frac{\varepsilon}{p}}$ is equal to $\frac{\Gamma\left(1+\beta-\frac{\varepsilon}{p}\right)}{\Gamma\left(1+\beta-\frac{\varepsilon}{p}-m\right)} x^{\beta-\frac{\varepsilon}{p}-m}$, it follows that

$$
\mathcal{D}_{+}^{m} \tilde{f}(x)=x^{\beta-\frac{\varepsilon}{p}-m} \cdot \chi_{(1, \infty)}(x) .
$$

Thus, the left-hand side of (21) may be bounded from above as follows:

$$
\begin{aligned}
\widetilde{R} & =C_{1}\left[\int_{1}^{\infty} x^{-1-\varepsilon} d x\right]^{\frac{1}{p}}\left[\sum_{n=1}^{\infty} n^{-1-\varepsilon}\right]^{\frac{1}{q}} \\
& <\frac{C_{1}}{\varepsilon^{\frac{1}{p}}}\left[1+\int_{1}^{\infty} x^{-1-\varepsilon} d x\right]^{\frac{1}{q}}=\frac{C_{1}(\varepsilon+1)^{\frac{1}{q}}}{\varepsilon} .
\end{aligned}
$$

On the other hand, utilizing the well-known Fubini theorem and a suitable change of variables, it follows that

$$
\begin{aligned}
\widetilde{L} & =\varphi(\varepsilon) \int_{1}^{\infty} x^{\beta-\frac{\varepsilon}{p}}\left(\sum_{n=1}^{\infty} K(x, n) n^{-p \alpha_{2}-\frac{\varepsilon}{q}}\right) d x \\
& >\varphi(\varepsilon) \int_{1}^{\infty} x^{-q \alpha_{1}-\frac{\varepsilon}{p}}\left(\int_{1}^{\infty} K(x, y) y^{-p \alpha_{2}-\frac{\varepsilon}{q}} d y\right) d x \\
& =\varphi(\varepsilon) \int_{1}^{\infty} x^{-\varepsilon-1} \int_{\frac{1}{x}}^{\infty} K(1, t) t^{-p \alpha_{2}-\frac{\varepsilon}{q}} d t d x \\
& =\frac{\varphi(\varepsilon)}{\varepsilon} \int_{1}^{\infty} K(1, t) t^{-p \alpha_{2}-\frac{\varepsilon}{q}} d t+\varphi(\varepsilon) \int_{1}^{\infty} x^{-\varepsilon-1} \int_{\frac{1}{x}}^{1} K(1, t) t^{-p \alpha_{2}-\frac{\varepsilon}{q}} d t d x \\
& =\frac{\varphi(\varepsilon)}{\varepsilon} \int_{1}^{\infty} K(1, t) t^{-p \alpha_{2}-\frac{\varepsilon}{q}} d t+\varphi(\varepsilon) \int_{0}^{1} K(1, t) t^{-p \alpha_{2}-\frac{\varepsilon}{q}} \int_{\frac{1}{t}}^{\infty} x^{-\varepsilon-1} d x d t \\
& =\frac{\varphi(\varepsilon)}{\varepsilon}\left(\int_{1}^{\infty} K(1, t) t^{-p \alpha_{2}-\frac{\varepsilon}{q}} d t+\int_{0}^{1} K(1, t) t^{-p \alpha_{2}+\frac{\varepsilon}{p}} d t\right),
\end{aligned}
$$

since the function $K(x, y) y^{-p \alpha_{2}}$ is decreasing on $\mathbb{R}_{+}$for any fixed $x \in \mathbb{R}_{+}$. Here, $\varphi$ stands for the function $\varphi(\varepsilon)=\frac{\Gamma\left(1+\beta-\frac{\varepsilon}{p}-m\right)}{\Gamma\left(1+\beta-\frac{\varepsilon}{p}\right)}$.

Now, relations (21), (22), and (23) entail the inequality

$$
\varphi(\varepsilon)\left(\int_{1}^{\infty} K(1, t) t^{-p \alpha_{2}-\frac{\varepsilon}{q}} d t+\int_{0}^{1} K(1, t) t^{-p \alpha_{2}+\frac{\varepsilon}{p}} d t\right)<C_{1}(\varepsilon+1)^{\frac{1}{q}} .
$$

Therefore, by the Fatou lemma, as $\varepsilon \rightarrow 0$, it follows that $L^{*} \cdot \frac{\Gamma(\beta-m+1)}{\Gamma(\beta+1)} \leq C_{1}$, which is in contrast to our assumption. Hence, $L^{*} \cdot \frac{\Gamma(\beta-m+1)}{\Gamma(\beta+1)}$ is the best constant in (17).

It remains to show that $L^{*} \cdot \frac{\Gamma(\beta-m+1)}{\Gamma(\beta+1)}$ is the best constant in (9). Similarly to the above discussion, suppose that there exists a positive constant $c_{1}$ smaller than $L^{*} \cdot \frac{\Gamma(\beta-m+1)}{\Gamma(\beta+1)}$ such that

$$
\left[\sum_{n=1}^{\infty} n^{(p-1)\left(1-p q \alpha_{2}\right)}\left(\int_{0}^{\infty} K(x, n) f(x) d x\right)^{p}\right]^{\frac{1}{p}}<c_{1}\left[\int_{0}^{\infty} x^{p(m-\beta)-1}\left(\mathcal{D}_{+}^{m} f(x)\right)^{p} d x\right]^{\frac{1}{p}}
$$


holds for all non-negative functions $f \in \Lambda_{+}^{m}$. Then, utilizing the well-known Hölder inequality, we have

$$
\begin{aligned}
& \sum_{n=1}^{\infty} a_{n} \int_{0}^{\infty} K(x, n) f(x) d x \\
& \quad=\sum_{n=1}^{\infty}\left[n^{\frac{1-p q \alpha_{2}}{q}} \int_{0}^{\infty} K(x, n) f(x) d x\right] \cdot\left[n^{\frac{-1+p q \alpha_{2}}{q}} a_{n}\right] \\
& \quad \leq\left[\sum_{n=1}^{\infty} n^{(p-1)\left(1-p q \alpha_{2}\right)}\left(\int_{0}^{\infty} K(x, n) f(x) d x\right)^{p}\right]^{\frac{1}{p}}\left[\sum_{n=1}^{\infty} n^{-1+p q \alpha_{2}} a_{n}^{q}\right]^{\frac{1}{q}} \\
& \quad<c_{1}\left[\int_{0}^{\infty} x^{p(m-\beta)-1}\left(\mathcal{D}_{+}^{m} f(x)\right)^{p} d x\right]^{\frac{1}{p}}\left[\sum_{n=1}^{\infty} n^{-1+p q \alpha_{2}} a_{n}^{q}\right]^{\frac{1}{q}}
\end{aligned}
$$

which leads to the result that $L^{*} \cdot \frac{\Gamma(\beta-m+1)}{\Gamma(\beta+1)}$ is not the best possible constant in (17). With this contradiction, the proof is completed.

Theorem 4 Let $p, q>1$ be conjugate parameters and $K: \mathbb{R}_{+} \times \mathbb{R}_{+} \rightarrow \mathbb{R}$ be a non-negative measurable homogeneous function of degree $-s, 0<s \leq 1$. Further, let $\alpha_{1}$ and $\alpha_{2}$ be real parameters fulfilling condition (16) and $\beta=-q \alpha_{1} \in(-1, s-1)$. If the function $K(x, y) y^{-p \alpha_{2}}$ is decreasing on $\mathbb{R}_{+}$for any fixed $x \in \mathbb{R}_{+}$, then $L^{*} \cdot \frac{\Gamma(-\beta)}{\Gamma(-\beta+m)}$ is the best constant in (19) and (20).

Proof We follow the lines of the proof of Theorem 3, that is, we assume that the inequality

$$
\begin{aligned}
& \sum_{n=1}^{\infty} a_{n} \int_{0}^{\infty} K(x, n) f(x) d x \\
& \quad=\int_{0}^{\infty} f(x)\left(\sum_{n=1}^{\infty} K(x, n) a_{n}\right) d x \\
& \quad<C_{2}\left[\int_{0}^{\infty} x^{p(m-\beta)-1}\left(\mathcal{D}_{ \pm}^{m} f(x)\right)^{p} d x\right]^{\frac{1}{p}}\left[\sum_{n=1}^{\infty} n^{-1+p q \alpha_{2}} a_{n}^{q}\right]^{\frac{1}{q}}
\end{aligned}
$$

holds with a positive constant $C_{2}$, smaller than $L^{*} \cdot \frac{\Gamma(-\beta)}{\Gamma(-\beta+m)}$. Now, let $\widetilde{L}$ and $\widetilde{R}$, respectively, denote the left-hand side and the right-hand side of inequality (25) accompanied by

$$
\tilde{f}^{*}(x)=\frac{\Gamma\left(-\beta+\frac{\varepsilon}{p}\right)}{\Gamma\left(m-\beta+\frac{\varepsilon}{p}\right)} x^{\beta-\frac{\varepsilon}{p}} \cdot \chi_{[1, \infty)}(x) \quad \text { and } \quad \tilde{a}_{n}^{*}=n^{-p \alpha_{2}-\frac{\varepsilon}{q}}
$$

where $\varepsilon>0$ is a sufficiently small number. Then, taking into account (23), we have

$$
\widetilde{L}>\frac{\varphi^{*}(\varepsilon)}{\varepsilon}\left(\int_{1}^{\infty} K(1, t) t^{-p \alpha_{2}-\frac{\varepsilon}{q}} d t+\int_{0}^{1} K(1, t) t^{-p \alpha_{2}+\frac{\varepsilon}{p}} d t\right),
$$

where $\varphi^{*}(\varepsilon)=\frac{\Gamma\left(-\beta+\frac{\varepsilon}{p}\right)}{\Gamma\left(m-\beta+\frac{\varepsilon}{p}\right)}$. 
On the other hand, since the $m$ th derivative of the function $x^{\beta-\frac{\varepsilon}{p}}$ is equal to $(-1)^{m} \times$ $\frac{\Gamma\left(m-\beta+\frac{\varepsilon}{p}\right)}{\Gamma\left(-\beta+\frac{\varepsilon}{p}\right)} x^{\beta-\frac{\varepsilon}{p}-m}$, it follows that $\mathcal{D}_{ \pm}^{m} \tilde{f}(x)=x^{\beta-\frac{\varepsilon}{p}-m} \cdot \chi_{(1, \infty)}(x)$, and so

$$
\widetilde{R}<\frac{C_{2}(\varepsilon+1)^{\frac{1}{q}}}{\varepsilon} .
$$

Now, comparing (25), (26), and (27), it follows that

$$
\varphi^{*}(\varepsilon)\left(\int_{1}^{\infty} K(1, t) t^{-p \alpha_{2}-\frac{\varepsilon}{q}} d t+\int_{0}^{1} K(1, t) t^{-p \alpha_{2}+\frac{\varepsilon}{p}} d t\right)<C_{2}(\varepsilon+1)^{\frac{1}{q}},
$$

and consequently, $L^{*} \cdot \frac{\Gamma(-\beta)}{\Gamma(-\beta+m)} \leq C_{2}$, after letting $\varepsilon \rightarrow 0$. This means that the constant $L^{*} \cdot \frac{\Gamma(-\beta)}{\Gamma(-\beta+m)}$ is the best possible in (19).

To conclude the proof, we suppose that, contrary to our claim, there exists a constant $0<c_{2}<L^{*} \cdot \frac{\Gamma(-\beta)}{\Gamma(-\beta+m)}$ such that the inequality

$$
\left[\sum_{n=1}^{\infty} n^{(p-1)\left(1-p q \alpha_{2}\right)}\left(\int_{0}^{\infty} K(x, n) f(x) d x\right)^{p}\right]^{\frac{1}{p}}<c_{2}\left[\int_{0}^{\infty} x^{p(m-\beta)-1}\left(\mathcal{D}_{ \pm}^{m} f(x)\right)^{p} d x\right]^{\frac{1}{p}}
$$

holds for all non-negative functions $f \in \Lambda_{ \pm}^{m}$, as in the statement of theorem. Finally, employing the Hölder inequality, we obtain

$$
\begin{aligned}
& \sum_{n=1}^{\infty} a_{n} \int_{0}^{\infty} K(x, n) f(x) d x \\
& \quad=\sum_{n=1}^{\infty}\left[n^{\frac{1-p q \alpha_{2}}{q}} \int_{0}^{\infty} K(x, n) f(x) d x\right] \cdot\left[n^{\frac{-1+p q \alpha_{2}}{q}} a_{n}\right] \\
& \quad \leq\left[\sum_{n=1}^{\infty} n^{(p-1)\left(1-p q \alpha_{2}\right)}\left(\int_{0}^{\infty} K(x, n) f(x) d x\right)^{p}\right]^{\frac{1}{p}}\left[\sum_{n=1}^{\infty} n^{-1+p q \alpha_{2}} a_{n}^{q}\right]^{\frac{1}{q}} \\
& \quad<c_{2}\left[\int_{0}^{\infty} x^{p(m-\beta)-1}\left(\mathcal{D}_{ \pm}^{m} f(x)\right)^{p} d x\right]^{\frac{1}{p}}\left[\sum_{n=1}^{\infty} n^{-1+p q \alpha_{2}} a_{n}^{q}\right]^{\frac{1}{q}},
\end{aligned}
$$

which is impossible since $L^{*} \cdot \frac{\Gamma(-\beta)}{\Gamma(-\beta+m)}$ is the best constant in (19). With this contradiction, the proof is completed.

Competing interests

The authors declare that they have no competing interests.

Authors' contributions

All authors participated in the design of this work and performed equally. All authors read and approved the final manuscript.

\section{Author details}

${ }^{1}$ Department of Mathematical Analysis, National University of Mongolia, P.O. Box 46A/125, Ulaanbaatar, 14201, Mongolia. ${ }^{2}$ Institute of Mathematics, National University of Mongolia, P.O. Box 46A/104, Ulaanbaatar, 14201, Mongolia. ${ }^{3}$ Faculty of Electrical Engineering and Computing, University of Zagreb, Unska 3, Zagreb, 10000, Croatia. 


\section{References}

1. Azar, LE: Two new forms of half-discrete Hilbert inequality. J. Egypt. Math. Soc. (2013). doi:10.1016/j.joems.2013.06.014

2. Hardy, GH, Littlewood, JE, Pólya, G: Inequalities, 2nd edn. Cambridge University Press, Cambridge (1967)

3. Krnić, M, Pečarić, J, Vuković, P: A unified treatment of half-discrete Hilbert-type inequalities with a homogeneous kernel. Mediterr. J. Math. 10, 1697-1716 (2013)

4. Huang, Q, Yang, B: On a more accurate half-discrete Hilbert's inequality. J. Inequal. Appl. 2012,106 (2012)

5. Krnić, M, Vuković, P: On a multidimensional version of the Hilbert type inequality. Anal. Math. 38, 291-303 (2012)

6. Rassias, MT, Yang, B: On half-discrete Hilbert's inequality. Appl. Math. Comput. 220, 75-93 (2013)

7. Yang, B, Krnić, M: A half-discrete Hilbert-type inequality with a general homogeneous kernel of degree 0. J. Math. Inequal. 6, 401-417 (2012)

8. Yang, B: A half-discrete Hilbert-type inequality with a non-homogeneous kernel and two variables. Mediterr. J. Math. 10, 677-692 (2013)

9. Krnić, M, Pečarić, J, Perić, I, Vuković, P: Recent Advances in Hilbert-Type Inequalities. Element, Zagreb (2012)

10. Abramowitz, M, Stegun, IA: Handbook of Mathematical Functions with Formulas, Graphs, and Mathematical Tables, 4th edn. National Bureau of Standards Applied Math. Series, vol. 55. U.S. Government Printing Office, Washington (1965)

11. Graham, RL, Knuth, DE, Patashnik, D: Concrete Mathematics - A Foundation for Computer Science. Addison-Wesley, Reading (1989)

12. Hardy, GH: Note on some points in the integral calculus, LXIV. Messenger Math. 57, 12-16 (1928)

13. Kufner, A, Maligranda, L, Persson, LE: The Hardy Inequality - About Its History and Some Related Results. Vydavatelský Servis, Pilsen (2007)

10.1186/1029-242X-2014-83

Cite this article as: Adiyasuren et al.: Half-discrete Hilbert-type inequalities involving differential operators. Journal of Inequalities and Applications 2014, 2014:83

\section{Submit your manuscript to a SpringerOpen ${ }^{\ominus}$ journal and benefit from:}

- Convenient online submission

- Rigorous peer review

- Immediate publication on acceptance

Open access: articles freely available online

- High visibility within the field

- Retaining the copyright to your article 